\title{
CORRELACIÓN DE VARIABLES MACROCLIMÁTICAS DEL OCÉANO PACÍFICO CON LOS CAUDALES EN LOS RÍOS INTERANDINOS DEL VALLE DEL CAUCA (COLOMBIA)
}

\author{
CORRELATION OF MACROCLIMATIC VARIABLES OF PACIFIC OCEAN WITH THE \\ VOLUMES OF INTERANDEAN RIVERS OF THE CAUCA VALLEY (COLOMBIA)
}

\author{
Yesid Carvajal ${ }^{1}$, Claudia Grisales ${ }^{2}$ y Julián Mateus ${ }^{2}$
}

\section{RESUMEN}

Los fenómenos climáticos sobre los océanos ejercen gran influencia sobre la hidroclimatologia del valle del rio Cauca (Colombia), en especial el evento "EI Niño Oscilación Sur" (ENOS), que es responsable de la variabilidad climática en escalas de tiempo que van desde meses hasta décadas. Debido a esta dependencia, se estudió la correlación entre 8 variables indicadoras del clima en los Océanos Pacifico y Atlántico con los caudales medios mensuales de 8 ríos del valle del río Cauca. Las series cubren un período promedio de 40 años 1957-1997. Los resultados muestran las variaciones regionales de la situación hidrológica del valle del río Cauca respecto a las variables macroclimáticas. Los coeficientes de correlación indican que las variables que presentan mayor asociación con los caudales analizados son en su orden: Las temperaturas de la superticie del mar en las regiones Niño 3, Niño 3-4, Niño 4 y SOI, mientras en el Atlántico, las mejores correlaciones las presentan: la Temperatura en el Trópico, en el Atlántico Norte (NATL) y Atlántico Sur (SATL) respectivamente.

Palabras claves: Modelos de pronóstico, ríos interandinos, EI Niño Oscilación Sur, valle del Cauca, Colombia.

\begin{abstract}
The climate phenomena on the ocean influence greatly on the hydroclimatology of the Valley of the Cauca River (Colombia); especially "EI Niño Southern Oscillation" event (ENSO); which is the cause of the climatic variability in time scales that go from months until decades. Due to this dependence, the correlation between 8 indicative variables of the climate both, in the Pacific and Atlantic Oceans with the mean monthly flow of 8 rivers from the Valley of the Cauca River were studied. The series cover a period average of 40 years 1957-1997. The results show the regional variations of the hydrological situation of the Valley of the Cauca Piver concerning the macroclimatic variables. The correlation coefficients indicate that the variables that introduce best association with the analyzed flows were in order: sea surface temperature of the Niño 3, Niño 3-4, Niño 4 regions and SOI, in the Atlantic, the best correlations were: the temperalure in the Tropic, in the North Atlantic (NATL) and South Atlantic (SATL) respectively.
\end{abstract}

Key words: Forecast models, Interandean rivers, El Niño Southern Oscillation, Cauca Valley, Colombia.

\section{INTRODUCCIÓN}

La variabilidad del clima incide sobre las condiciones socioeconómicas de todos los habitantes del planeta, por lo cual, su

1 Ingeniero Agricola. M.Sc. Profesor Area Hidrologia-Riegos y Drenajes. Fac. de Ingeniería. Univ. del Valle

E-mail: yecarvaj@mafalda.univalle.edu.co

2 Ingeniero Agrícola. Univ. del Valle entendimiento permite prevenir y mitigar efectos adversos, así como obtener mejores predicciones. El régimen hidroclimático de Colombia está determinado localmente, por la presencia de costas sobre dos mares, la fisiografía, la circulación de la cuenca Amazónica y la vegetación entre otros, mientras que a nivel global, está determinada por la oscilación meridional de la Zona de Convergencia Intertropical, que está 
directamente relacionada con la ocurencia del evento "EI Niño Oscilación Sur" (ENOS), el cual es un calentamiento anormal de la temperatura del Océano Pacífico Ecuatotial, cerca de las costas de Ecuador y Perú, que se propaga a través de la cuenca oceánica, y que por debilitamiento de los vientos Alisios del este produce una profundización de la termoclina. Con el presente estudio, el grupo de Hidrología - Riegos y Drenajes ha iniciado el análisis de la dependencia de la hidroclimatología de la región con diferentes variables macroclimáticas, buscando como objetivo final, involucrar las más simnificativas en los modelos de pronóstico de las diferentes fuentes hídricas.

\section{MARCO DE REFERENCIA}

La Oscilación Sur es un indicativo de la Circulación Ecuatorial que esta ligada a las variaciones climáticas en todo el planctat (Glantz et al., 1991). Esta caracterizada por un gradiente en las presiones superficiales entre el este y el oeste del Océano Pacífico Ecuatorial. Generalmente. un centro de alta presión se localiza en el Pacilico sur cerca de Tahití ( $18^{\circ}$ LS, 150 L.W), mentras que un centro de baja presión se focaliza en Indonesia y el norte de Australia, D. rwin (12" LS, 131 LE). El gradicnte de presiones se expresa mediante el Indice de Oscilacion Sur (SOH), que se define como la diferencia de las presiones atmostéricas cutre esas do estaciones. Las anomalias negalivas del SOI están asociadas a la ocumencia de crentos cálidos de "El Niño", y las positivas con los eventos fríos de "La Niña" (Philander. 1991 ).

Mesa et al. (1997) confirman que en Latinoamérica hay referencias do perturbaciones climáticas asocialas al ENOS que indican su influencia en escalas de tiempo que van desde meses a años, entre las que se destacan: México (Cavazos y Hastenrath, 1990), América Central y Caribe (Rogers. 1988), Colombia, (Mesa et al., 1997). Venezuela, (Pulwarty el al., 1992), Perú (Waylen y Caviedes, 1986). en la cuenca
Amazónica (Marengo, 1992), (Marengo y Hastenrath, 1993) (Obregón y Nobre, 1990), Brasil (Kousky et al., 1984; Rao y Hada, 1990; Hastenrath y Greischar, 1993; Kayano et al., 1988; Chu, 1991), Sureste de Sudamérica (Mechoso y Pérez, 1992), América del Sur (Aceituno, 1988 y 1989).

Durante las lases extremas del ENOS, se presenta un desplazamiento del centro de Convección dentro de la llamada Zona de Convergencia Intertropical hacia el sur y hacia el oeste (Pulwarty y Díaz, 1993), ocasionado por el calentamicnto de las aguas frías del Océano Pacífico fuera de las costas del Perú y Ecuador, y por los cambios del gradiente de temperatura entre la tierra y el océano en la región noroccidental de Sudamérica. Poveda y Mexa " $a l .(1997)$ mencionan que otro factor que pucde influir en las anomalias hidrológicas cs la alteración del gradicnte de temperaturas del Océano Pacifico entre las costas de Colombia y Perú, que perturba la advección de aire lrío y húmedo desde el océano hacia el interior de Colombia; también mencionan la retroalimentación positiva que los extremos hidrológicos tienen sobre si mismos: condiciones de sequía ocasionadas por un forzamiento de macroescala disminuyen la precipitación, y a su vez la humedad del suelo y la evapotranspiración, por lo cual habrá menor disponibilidad de humedad para la convección atmosférica.

La Oscilacion del Atantico Norte (NAO), us lalternancia de la masa atmosférica entre las regiones subtropical y subpolar del Océano Atlántico. Al igual que el ENOS, la NAO se define como la variabilidad de la presión en sitios claves. Un centro está en las islas Azores, bajo la influencia del centro de alta presión subtropical y el otro sobre Akureyri (Islandia). en donde predominan bajas presiones atmosféricas a nivel del mar durante todo el año. La NAO se caracteriza por variaciones en el gradiente de presiones a nivel del mar en escalas mensual y estacional. Las correlaciones entre el ENOS y la NAO son muy bajas indicando que son débilmente 
dependientes, lo que confirma el resultado de Rogers (1984) citado por Mesa et al. (1997).

\section{METODOLOGÍA}

Las variables climáticas del Océano Pacífico que se trabajaron fueron: Índice de Oscilación Sur, SOI, temperatura superficial del mar en las regiones Niño 1-2 (0-10 LS, $\left.90-80^{\circ} \mathrm{LW}\right)$, Niño $3\left(5^{\circ} \mathrm{LN}-5^{\circ} \mathrm{LS}, 150-90^{\circ}\right.$ LW), Niño $4\left(5^{\circ} \mathrm{LN}-5^{\circ} \mathrm{LS}, 160^{\circ} \mathrm{LE}-150^{\circ}\right.$ LW), y Niño 3-4 los cuales se denominaran SST (Fig. 1). Del Océano Atlántico se trabajó con: las temperaturas en el Atlántico Norte (NATL), temperaturas en el Atlántico Sur (SATL), la temperatura en la región tropical del Atlántico (TROP). Esta información fue obtenida del Boletín del Climate Analysis Center que distribuye la NOAA (National Oceanic Atmospheric Administration) por internet.

Se efectuó un análisis descriptivo, calculando los principales estadísticos de cada serie, como son la media, desviación estándar, valor máximo, mínimo y el coeficiente de variación. Seguidamente, se efectuó correlaciones entre el caudal y cada variable, desfasándola desde 0 hasta 12 meses (variable macroclimáticas rezagada $t$ meses frente al caudal). Posteriormente, se calculó la autocorrelación del caudal, para hallar su asociación con los caudales antecedentes, que generalmente brindan una alta capacidad de predicción, dada su alta persistencia hidrológica. Las variables se suavizaron mediante un promedio móvil de tres meses, con el objeto de filtrar la variabilidad de alta frecuencia, tratando de preservar al máximo la señal original, para finalmente, estimar los coeficientes de correlación, con las series filtradas.

\section{RESULTADOS}

Para el río Cauca, estación Juanchito, el mayor grado de asociación lo presentaron las variables NIÑO 3 desfasada 4 meses, NIÑO 3-4 desfasada 3 meses, TROP desfasada 4 meses y $Q_{.1}$ desfasado un mes, convirtiéndose en las que potencialmente aportan mayor información para el pronóstico del caudal. Los

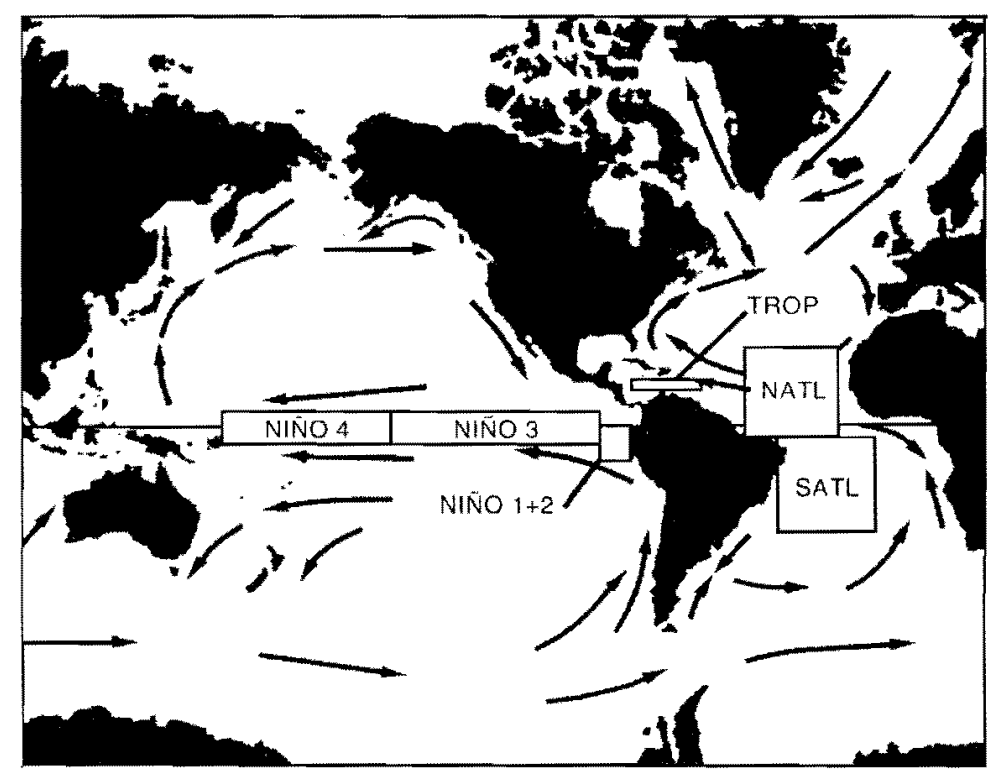

Figura 1. Localización de los bloques de medición de la temperatura superficial del mar Niño $1+2$, Niño 3 y Niño 4. 
correlogramas de caudales vs. variables macroclimáticas para diferentes rezagos se presentan en la Fig. 2.

Los valores de las mejores correlaciones muestran claramente, la influencia de las temperaturas del Océano Pacífico sobre la hidrología de la región (Tab, 1). Mesa et al. (1997) informan que las mayores correlaciones del SOI y los registros de caudales de Colombia se presentan en la región occidental y la zona andina, mientras que los ríos sobre la costa del Mar Caribe están más afectados por fenómenos océano atmosféricos que ocurren en el Atlántico y el Mar Caribe, como en el caso de la NAO. Con excepción de los ríos del Caribe, la gran mayoría de los ríos de Colombia presentan el mayor coeficiente de correlación para rezagos positivos, es decir para los rezagos en los cuales la hidrología precede a la NAO, pareciendo confirmar la hipótesis sobre la interacción que estaría ejerciendo la hidroclimatología continental del norte de Sudamérica sobre el Caribe y el Atlántico (Poveda y Mesa, 1996).

Aunque los valores de las correlaciones para rezagos negativos (NAO) (antecediendo la hidrología) no son muy altos, tampoco son despreciables, y pueden ser de utilidad para la predicción de la hidrología en la región, si se tiene en cuenta que el SOI y la NAO parecen ser no dependientes.

\section{Calibración de Modelos de Pronóstico}

Para la calibración del modelo de pronóstico se empleó la herramienta de Regresión Lineal Múltiple, porque permite incluir un mayor número de variables explicativas potenciales. De acuerdo con los resultados obtenidos de las correlaciones entre caudales y variables macroclimáticas, se consideró como predictores (variables explicativas de caudal) todas la variables macroclimáticas rezagadas hasta 12 meses y adicionalmente, los 2 primeros rezagos de la variable caudal, debido a que guardan fuerte relación con los inmediatamente anteriores (persistencia).

Algunos autores sugieren seleccionar como potencial predictor únicamente el rezago que tenga mayor valor de correlación con el caudal. Sin embargo, se tiene la limitante que al seleccionar sólo la variable rezagada con mayor correlación, se eliminan otros rezagos estadísticos significativos. Adicionalmente, para este caso, las variables macroclimáticas estudiadas no son independientes entre sí, por lo que puede ocurrir que una variable sea poco significativa individualmente, pero asociada con otras sí lo sea. Por tal motivo, se utilizaron todas las variables macroclimáticas con el mayor número posible de rezagos, para construir el modelo de regresión, una vez seleccionados los mejores predictores.

Se estimaron los coeficientes de regresión

Tabla 1. Mejores correlaciones entre los caudales del río Cauca (Juanchito) y las variables macroclimáticas.

\begin{tabular}{cccccc}
\hline $\begin{array}{c}\text { Variable } \\
\text { macroclimática }\end{array}$ & $\begin{array}{c}\text { Correlación } \\
\text { con caudal o }\end{array}$ & $\begin{array}{c}\text { Rezago } \\
\text { (meses) }\end{array}$ & $\begin{array}{c}\text { Variable } \\
\text { macroclimática }\end{array}$ & $\begin{array}{c}\text { Correlación } \\
\text { con caudal }\end{array}$ & $\begin{array}{c}\text { Rezago } \\
\text { (meses) }\end{array}$ \\
\hline SOI & 0,42 & 0 & NATL & 0,42 & 10 \\
SST 1-2 & 0,59 & 5 & SATL & 0,42 & 10 \\
SST 3 & 0,65 & 4 & TROP & 0,65 & 4 \\
SST 3-4 & 0,65 & 3 & & & \\
SST 4 & 0,59 & 2 & & & \\
\hline Autocorrelación & Correlación & & & & \\
caudales & con caudal o & & & & \\
\hline Q-1 & 0,65 & 1 & & & \\
Q-2 & 0,27 & 2 &
\end{tabular}



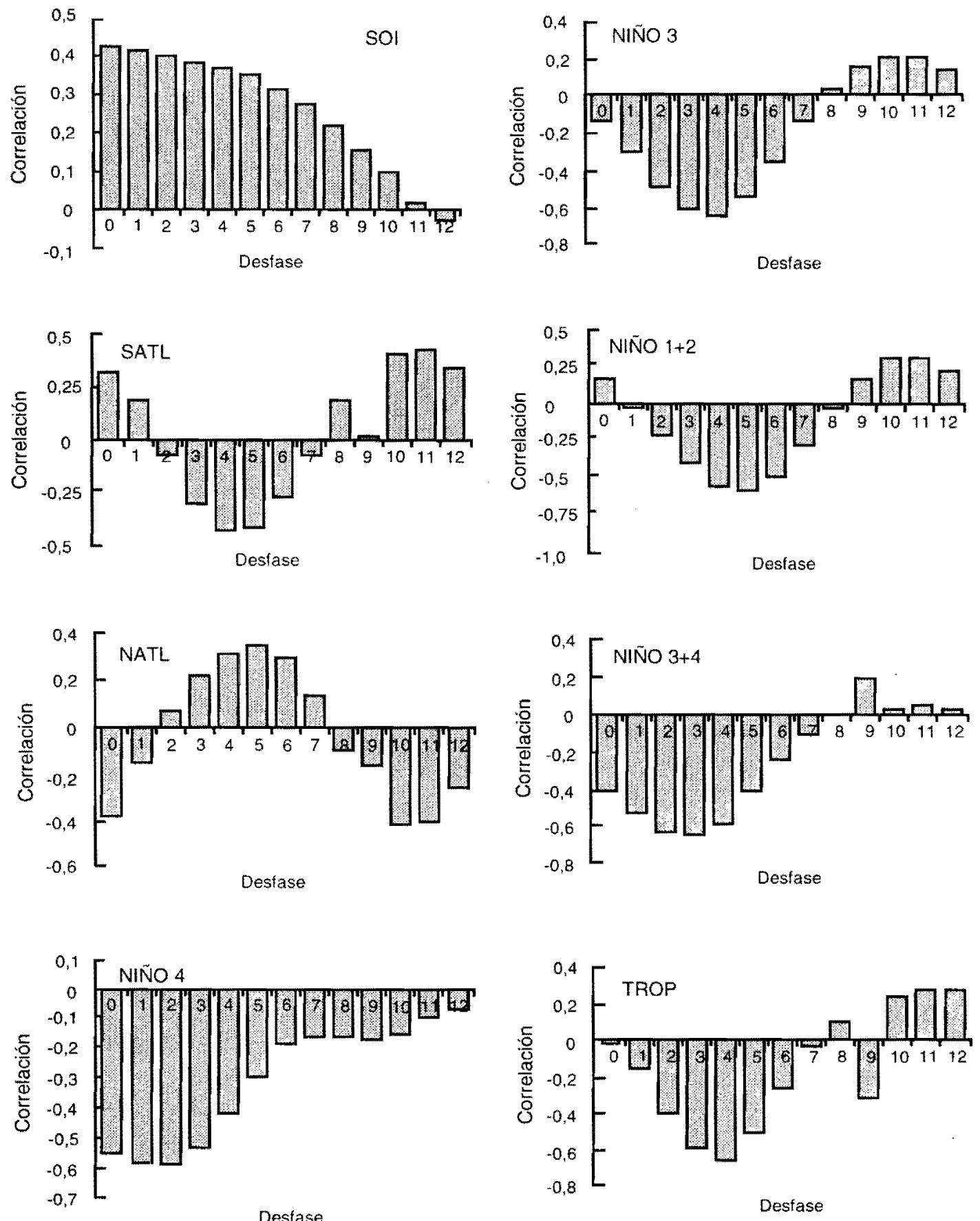

Figura 2. Comportamiento de los coeficientes de correlación múltiple entre los caudales medios mensuales de la estación Juanchito (río Cauca) y las variables macroclimáticas para diferentes rezagos. 
Tabla 2. Validación del modelo para caudales medios mensuales en el río Cauca (Juanchito).

\begin{tabular}{lcccc}
\hline \multicolumn{1}{c}{ Predictor } & Coeficiente & D.E. & $t$ & $p$ \\
\hline Constante & 0,0087 & 0,014 & $-0,62$ & 0,54 \\
$Q_{\mathrm{t}-1}$ & 1,295 & 0,036 & 36,23 & 0,00 \\
$\mathrm{Q}_{\mathrm{t}-2}$ & $-0,7331$ & 0,033 & $-22,28$ & 0,00 \\
SST 1-2 & $-0,1871$ & 0,032 & $-5,80$ & 0,00 \\
SST 4 & $-0,2065$ & 0,021 & $-9,97$ & 0,00 \\
TROP & 0,1336 & 0,037 & 3,60 & 0,00 \\
\hline
\end{tabular}

\section{Análisis de varianza}

\begin{tabular}{lccccc}
\hline & GL & SC & Var & F & Valor Crít. F \\
\hline Varianza explicada & 5 & 296 & 59,3 & 650,28 & 0,00 \\
Varianza residual & & 467 & 42 & 0,09 & \\
\hline Total & 472 & 339 & & & \\
\hline & & & & Var: Varianza \\
t: Estadístico & Gl: Grados de libertad & & F: Estadistico &
\end{tabular}

y se validó el modelo obtenido. La validación de un modelo de predicción exige alta rigurosidad, para lo cual existen muchos métodos que prueban las hipótesis bajo las cuales se desarrolló el modelo lineal, así como la significancia y capacidad predictiva del mismo. Para este caso, se trabajó con pruebas que se consideran de mayor relevancia de acuerdo con los objetivos perseguidos. Una de las primeras pruebas que recomienda la literatura para validación de un modelo, es el contraste entre los coeficientes de regresión, tanto a nivel individual como en conjunto. Para el constraste individual se utilizó el estadístico $t$, con el que se determinó si la variable influye en la respuesta. Para esto, el estadístico debe ser mayor o igual a un número de referencia. Para el caso, se escogió 1,96 que corresponde a un nivel de significancia de 0,05 (Tab. 2).

Para evaluar la significancia de las variables en conjunto, se aplicó el estadístico $\mathrm{F}$, determinándose como significativo si el nivel obtenido era menor o igual a 0,05 . Todos los modelos pasaron la prueba. La Tab. 2 presenta la validación del modelo final hallado para pronósticos de los caudales del río Cauca con un mes de anticipación, incluyendo los contrastes sobre los coeficientes de regresión y la varianza explicada por la regresión.

Para el caso del río Cauca en la Estación Juanchito, se encontró el siguiente modelo:

$Q_{\text {ext }}=-0,0087+1,294 Q_{t-1}-0,7331 Q_{1-2}-$ $0,1871 \mathrm{SST}_{1.2}-0,2066 \mathrm{SST}_{4}+0,1336 \mathrm{TROP}^{-13}$

Cuyos estadísticos asociados son:

$$
\begin{array}{ll}
\mathrm{S}=0,3021 & \mathrm{R}^{2}=87,44 \% \\
\mathrm{R}_{\text {cor }}^{2}=87,30 \% & \mathrm{r}=93,51 \%
\end{array}
$$

Donde $S$ es la desviación residual, $R^{2}$ es el coeficiente de determinación de la regresión, $\mathrm{R}^{2}{ }_{\text {corr }}$ es el coeficiente de determinación corregido por grados de libertad y $\mathrm{r}$ es el coeficiente de correlación. Es importante disponer del $\mathrm{R}^{2}$ corregido como criterio para evaluar globalmente un modelo de correlación lineal, pues el coeficiente de correlación no corregido se incrementa aunque en el modelo se incluya variables no representativas, mientras el corregido castiga el $\mathrm{R}^{2}$ por el número de parámetros del modelo.

De igual forma se determinó un modelo sin tener en cuenta los caudales antecedentes, 
con el fin de expresarlo sólo en función de las variables macroclimáticas, encontrándose que las variables Niño 3 y SOI adquieren significancia. Sin embargo, el coeficiente de correlación sólo alcanza el valor de $71,9 \%, y$ una desviación residual de 0,5922 , mientras que utilizando únicamente los caudales antecedentes, se obtuvo un modelo con coeficiente de correlación de $91,3 \%$ y 0,345 de desviación residual. Lo anterior indica que el mejor modelo se obtiene de involucrar la combinación de las variables regresivas y las macroclimáticas; así, se reduce la desviación residual a 0,3021 y se mejora la correlación a $93,5 \%$.

La Tab. 3 presenta los coeficientes de correlación individual obtenidos entre las variables más significativas y los caudales medios mensuales de ríos del valle del río Cauca. Se destaca la gran influencia de los caudales antecedentes $\left(Q_{t-1}\right.$ y $\left.Q_{t-2}\right)$, siendo las variables que mayor información aportan.

Mesa et al. (1997) señalan que la influencia del ENSO es más evidente en el occidente y suroccidente del país, con menor influencia en la Costa Caribe y en las estaciones de la Cordillera Oriental que pertenecen a la Amazonía y Orinoquía. El análisis de correlación a diferentes rezagos $(-12$ meses a 12 meses), que no se presenta aquí, demuestra que entre 3 y 4 meses se presentan las mayores correlaciones, lo cual indica una gran ayuda para los modelos de predicción.

\section{Ajuste del modelo de pronóstico vs. esperado}

Para juzgar la validez de los modelos se utilizó la comparación entre los pronósticos y las ocurrencias históricas, usando para esto un período no utilizado para estimar el modelo. En este trabajo, la validación se realizó utilizando el período enero de 1991 a diciembre de 1997 (Fig. 3).

El ajuste entre caudales observados y pronosticados se puede calificar como satisfactorio, lo cual era de esperarse debido al buen ajuste del modelo (coeficiente de correlación) y a la alta persistencia de los caudales. Los modelos en general, capturan las señales de baja o aumento de los caudales. lo cual es particularmente importante ya que permite determinar con anticipación, la disminución o aumento de los mismos. Para juzgar la validez de la comparación entre lo real y lo pronosticado se requiere asociar el pronóstico a bandas de confianza, para calificar el desempeño del modelo en términos de los aciertos en el pronóstico.

\section{CONCLUSIONES Y RECOMENDACIONES}

Las variables macroclimáticas relacionadas con TSS, resultan significativas en la explicación de nuestros caudales. El resultado anterior era de esperarse y está acorde con resultados de Mesa et al. (1997). Al involucrar

Tabla 3. Coeficientes de correlación entre los caudales medios mensuales y las variables más significativas.

\begin{tabular}{lcccccccccc}
\hline Rio & $\mathbf{Q}_{\mathrm{t}-1}$ & $\mathbf{Q}_{\mathrm{t}-2}$ & SOI & Niño 1+2 & Niño 3 & Niño 4 & Niño 3-4 & NATL & SATL & TROP \\
\hline Cauca (Juanchito) & 0,647 & 0,264 & 0,424 & $-0,588$ & $-0,650$ & $-0,585$ & $-0,650$ & $-0,425$ & 0,422 & $-0,647$ \\
Jamundi (Potrerito) & 0,499 & & 0,342 & $-0,442$ & $-0,504$ & $-0,468$ & $-0,511$ & $-0,351$ & 0,341 & $-0,483$ \\
Timba (Timba) & 0,527 & & 0,352 & $-0,315$ & $-0,392$ & $-0,378$ & $-0,408$ & 0,152 & 0,192 & $-0,380$ \\
Cauca (La Victoria) & 0,679 & 0,294 & 0,455 & $-0,515$ & $-0,609$ & $-0,596$ & $-0,636$ & $-0,286$ & 0,208 & $-0,413$ \\
Ovejas (Ovejas) & 0,689 & 0,352 & 0,372 & $-0,652$ & $-0,659$ & $-0,536$ & $-0,600$ & 0,489 & $-0,516$ & $-0,642$ \\
Quinamayo (Ouinamayo) & 0,584 & 0,237 & 0,368 & $-0,528$ & $-0,500$ & $-0,534$ & $-0,579$ & $-0,344$ & $-0,379$ & $-0,569$ \\
Morales (Santa Librada) & 0,616 & 0,350 & 0,512 & $-0,303$ & $-0,440$ & $-0,579$ & $-0,513$ & $-0,188$ & $-0,140$ & $-0,487$ \\
La Vieja (Cartago) & 0,833 & 0,500 & 0,472 & $-0,500$ & $-0,594$ & $-0,570$ & $-0,625$ & $-0,364$ & 0,319 & $-0,588$ \\
Tulua (Mateguadua) & 0,597 & 0,253 & 0,507 & $-0,376$ & $-0,530$ & $-0,621$ & $-0,601$ & $-0,248$ & $-0,248$ & $-0,472$ \\
\hline
\end{tabular}




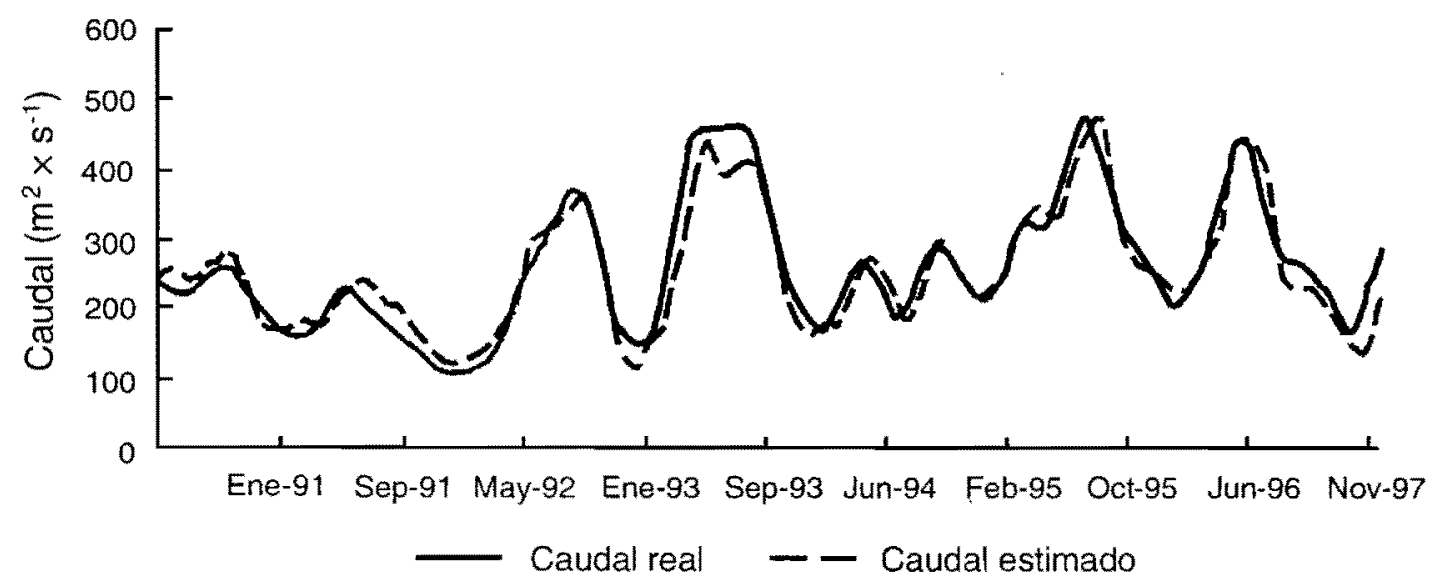

Figura 3. Modelo de pronóstico para los caudales del río Cauca, durante el período enero 91 - diciembre 97.

las variables autorregresivas, el SOI no resultó significativo para ningún río, pero al incluir sólo las variables macroclimáticas, adquiere significancia, lo que indica su incidencia en las variables autorregresivas. Las variables macroclimáticas que más relación tienen con la hidrología del valle del Cauca, son aquellas medidas en el Océano Pacífico, como las TSS en las regiones Niño 3-4 y Niño 3 para 3 y 4 meses de anticipación. Esto indica una gran ayuda para los modelos de predicción, cuando se involucra dichas variables, para las cuales es importante cuantificar el grado de dependencia lineal entre ellas a través de coeficientes de correlación a distintos rezagos.

La relación ENOS hidrología de la región no es simple ni lineal; es parte integral de un ciclo aperiódico en la interacción OcéanoAtmósfera-Tierra en la zona Ecuatorial, caracterizada por una escala espacial global y una baja frecuencia ( $2-5$ años), cuya principal manifestación es el ENOS. La relación indica que cuando el Pacífico está en una fase cálida se presenta en la región un clima más seco, mientras que la fase fría está acompañada de mayores lluvias (La Niña). Dicha relación permite regresiones lineales entre caudales y variables macroclimáticas indicadoras del fenómeno "El Niño", que generan ecuaciones que mejoran las predicciones que se pueden obtener con un modelo autorregresivo simple.

Teniendo en cuenta el carácter no lineal de los principales procesos atmosféricos, oceánicos e hidrológicos, en un futuro próximo se planteará el análisis con modelos no lineales de predicción, involucrando un mayor número de variables macroclimáticas. Aunque estos modelos de regresión lineal múltiple son bastante sencillos, se obtienen ecuaciones que mejoran las predicciones, sin embargo, es necesario tener en cuenta que los caudales mensuales de los meses anteriores al pronóstico presentan coeficientes de autocorrelación importantes, debido a la regulación que realiza la cuenca; esto amerita que dichas variables sean incluidas en los mismos.

\section{LITERATURA CITADA}

Accituno, P. 1988. On the functioning of the Southem Oscillation in the South American sector. Pant 1: Surface Climate. Mon. Wea. Rev. 116:505524.

-1989. On the functioning of the Southem Oscillation in the South American sector. Part II: Upper-air circulation. I. Climate 2: 341 355.

Cavazos. T. \& S. Hastenrath. 1990. Convection and rainfall over Mexico and their modulation by 
the Southern Oscillation. In. I. Chimatol. 10: 377-386.

Chu, P-S. 1991. Brazil's climate anomalies and ENSO. In: M. Glantz: R.K. Katz \& N. Nicholls (eds.). Teleconnections linking worldwide climate anomalies. Pp. 42-64.

Glantz. M.H.; R.K. Katz \& N. Nicholls (eds.). 1991. Teleconnections linking worldwide climate anonalies. Cambridge University Press, 535 pp.

Hastenrath. S. \& L. Greischar. 1993. Circulation mechanisms related to northeast Brazil rainfall anomalies. I. Geophys. Res 98: 5093-5102.

Kayano, M.T.; V.B. Rao \& A.D. Moura. 1988. Tropical circulation and the associated rainfall anomalies during two contrasting years. $J$. Climalol. 8: 477-488.

Kousky, V.E.: M.T. Kayano \& I.F.A. Cavlacanti. 1984. A revicw of the Southem Oscillation: Oceanicatmospheric circulartion changes and related rainfall anomalies. Tellus 36A: 490-504.

Marengo, 1. 1992. Interannual variability of surface climate in the Amazon basin. J. Climatol. 12: $853-863$

Marengo, J. \& S. Hastenrath. 1993. Case studies of extreme climatic events in the Amazon basin. J. Climate 6: $617-627$.

Mechoso, C.R. \& G. Perez. 1992. Streamflow in the southeastern South America and the Southern Oscillation. I. Climate 5: 1535-1539.

Mesa, O.; G. Poveda y L.F. Carvajal. 1997. Introducción al clina de Colombia. Imprenta Universidad Nacional de Colombia. Bogotá, Colombia. $390 \mathrm{pp}$.
Obregón, G.O. \& C.A. Nobre. 1990. Principal component analysis of precipitation fields over the Amazon River basin. Clinanalise 5: 3546.

Philander, G. 1991. El Niño, La Niña and the Southem Oscillation. Academic Press, 393 pp.

Poveda. G. y O. Mesa. 1996. Evidencia de Suramerica tropical como pucnte tierta atmósfera cntre los océanos Pacífico y Atlántico, Menoricts del IV Congreso Colombiano de Meteorologia. IDEAM, Santafé de Bogotá, pp. 178-187.

Pulwaty, R.S. \& H.F. Diaz. 1993. A study of the seasonal cycle and its perturbation by ENSO in the tropical Americas. IV Inemall. Confl. South. Hemisph. Met. Ocean, American Meteorological Society. Pp. 262-263.

Pulwarty, R.S.; R.G. Barry \& H. Riehl, 1992. Annual and seasonal patterns of raintall variability over Venezucla. Ertkunde 46: 273-280.

Rao, V.B. \& K. Hada. 1990. Characteristics of rainlal over Brazil: annual variations and connections with the Southern Oxcillation. Theor. Appl. Climatol. 42:81.91.

Rogers. J.C. 1984. The association between the North Atlantic Oscillation and the Southem Oscillation in the Northern Hemisphere. $M(n t$. Wea. Rev. 112: 1999-2015.

- 1988. Precipitation variability over the Caribbean and Tropical Americas associated with the Southern Oscillation. I. Clinate 1 : $172-182$.

Waylen, P.R. \& C.N. Caviedes. 1986. El Niño and annual floods on the north Peruvian litoral. $/$. Holrol. 89: 141.156 